\title{
Low Primary Cesarean Rate and High VBAC Rate With Good Outcomes in an Amish Birthing Center
}

\author{
James Deline, $M D^{1}$ \\ Lisa Varnes-Epstein, MHS, PA-C, \\ $C P M^{1}$ \\ Lee T. Dresang, $M D^{2}$ \\ Mark Gideonsen, $M D^{2}$ \\ Laura Lynch ${ }^{2}$ \\ Jobn J. Frey III, $M D^{2}$ \\ 'Amish Birthing Center, La Farge, \\ Wisconsin \\ ${ }^{2}$ University of Wisconsin School of \\ Medicine and Public Health, Madison, \\ Wisconsin
}

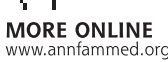

Conflicts of interest: The authors include the practitioners who founded and operate the birtbing center.

\section{CORRESPONDING AUTHOR}

Jim Deline, MD

La Farge Medical Clinic-VMH

$111 \mathrm{~W}$ Snow St

LaFarge, WI 54639

jdeline@vmh.org

\begin{abstract}
PURPOSE Recent national guidelines encourage a trial of labor after cesarean (TOLAC) as a means of increasing vaginal births after cesarean (VBACs) and decreasing the high US cesarean birth rate and its consequences (2010 National Institute of Health Consensus Statement and American College of Obstetricians and Gynecologists revised guideline). A birthing center serving Amish women in Southwestern Wisconsin offered an opportunity to look at the effects of local culture and practices that support vaginal birth and TOLAC. This study describes childbirth and perinatal outcomes during a 17-year period in LaFarge, Wisconsin.
\end{abstract}

METHODS We undertook a retrospective analysis of the records of all women admitted to the birth center in labor. Main outcome measures include rates of cesarean deliveries, TOLAC and VBAC deliveries, and perinatal outcomes for 927 deliveries between 1993 and 2010.

RESULTS The cesarean rate was 4\% (35 of 927), the TOLAC rate was $100 \%$, and the VBAC rate was $95 \%$ (88 of 92). There were no cases of uterine rupture and no maternal deaths. The neonatal death rate of 5.4 of 1,000 was comparable to that of Wisconsin $(4.6$ of 1,000) and the United States $(4.5$ of 1,000).

CONCLUSIONS Both the culture of the population served and a number of factors relating to the management of labor at the birthing center have affected the rates of cesarean delivery and TOLAC. The results of the LaFarge Amish study support a low-technology approach to delivery where good outcomes are achieved with low cesarean and high VBAC rates.

Ann Fam Med 2012;10:530-537. doi:10.1370/afm.1403.

\section{INTRODUCTION}

7 he cesarean rate in the United States has risen from 5.5\% in 1970 to $16.5 \%$ in $1980^{1}$ to $21 \%$ in 1996 to $32.9 \%$ in $2009 .{ }^{2,3} \mathrm{~A} 2011$ study calculates that if trends continue, the 2020 cesarean rate will be $56.2 \%{ }^{4}$ The 2007 primary cesarean rate was $23.4 \% .{ }^{5}$ The vaginal birth after cesarean (VBAC) rate-the percentage of pregnant women who give birth vaginally after a previous cesarean-has fallen from a high of $28.3 \%$ in $1996{ }^{1}$ to $8.5 \%$ in $2006 .{ }^{6}$ The World Health Organization (WHO) and Healthy People 2020 have suggested the ideal cesarean rate should be around $15 \%{ }^{7,8}$

The 2010 National Institutes of Health consensus conference on VBAC highlighted high-grade evidence that maternal mortality risk is decreased by VBAC compared with a repeat cesarean (3.8 vs 13.4 of 100,000$)$. $^{2}$ Data suggest decreasing the primary cesarean delivery rate and increasing the VBAC rate as key strategies to decrease the US cesarean rate. ${ }^{9}$

Amish communities ascribe religious and cultural value to childbearing. ${ }^{10,11}$ Contraception, including sterilization, may be prohibited. ${ }^{10}$ Advanced maternal age and grand multiparity are common. A study of 
6,623 deliveries in an Ohio Amish Community found that $24 \%$ of births were to women aged 35 years or older, and the median number of children for 475 women older than 44 years was 8.3. ${ }^{12}$ Health insurance of any kind and participation in such programs as Social Security, Medicare, and Medicaid are rare. ${ }^{10}$ Although generalizations can be made about Amish communities, each community is different. This study focused on a Southwest Wisconsin Amish community serviced by the LaFarge Birthing Center.

Amish women of Southwest Wisconsin generally give birth at home attended by an unlicensed birth attendant, mother, mother-in-law, or neighbor. Formal education for men and women is through 8 th grade, and women do not work outside the home. Amish culture prohibits electricity, telephone service, and car ownership, leading to delays in care for pregnancy complications. Responding to a community request, the LaFarge Medical Clinic developed a low-cost, community-based birthing center in the office staffed by a family physician and certified professional midwife to provide a safer alternative for Amish women. Registered nurses, midwifery interns, or trained laypeople with clinical skills also attended the births. This study describes a 17-year experience with Amish women giving birth in the LaFarge Birthing Center (a photograph of which is shown in Supplemental Figure 1, available

at http://annfammed.org/

\section{METHODS}

The LaFarge Birthing Center was developed as an alternative to home birth for Amish women. Originally, higher-risk patients were encouraged to give birth in the hospital. With time it was apparent that most higherrisk patients were having their babies at home rather than the hospital. After discussion of risks and benefits, these patients were increasingly allowed an attempted birthing center delivery. Patients were asked to review and sign a consent form contrasting treatments available at the birth center vs hospital, by which they agreed to abide by birth attendants' deci- sion for hospital transfer at any time. Women wanting a TOLAC were advised of uterine rupture risk, and verbal consent was obtained. Women not accepted for birthing center delivery included those with known placenta previa or brisk third-trimester bleeding, severe preeclampsia, nonfrank breech presentation not amenable to external version, and presentation of a first twin in a nonvertex position. Although birthing center prenatal care was encouraged, women who had planned home births could be considered for transfer to the birthing center before or during labor. Women evaluated in the birthing center and then sent to the hospital for reasons including birth center contraindications described above were included in birthing center statistics. Lafarge Birthing Center practices are outlined in Table $1 .{ }^{13}$ An ambulance service near the birthing center allowed prompt transport. At Vernon Memorial Hospital $(\mathrm{VMH}), 20$ minutes from the birthing center, births were attended by family physicians and midwives; a general surgeon performed cesareans. The VMH cesarean rate from 2006 through 2010 was $24.9 \%$.

\section{Table 1. LaFarge Birthing Center Practices}

\begin{tabular}{|c|c|}
\hline Condition & Management \\
\hline Previous cesarean & Trial of labor expected by women and supported by clinician \\
\hline $\begin{array}{l}\text { Breech or trans- } \\
\text { verse lie }\end{array}$ & $\begin{array}{l}\text { External cephalic version offered from } 37 \text { to } 38 \text { weeks' gestation; if } \\
\text { unsuccessful or declined, vaginal breech delivery offered if frank } \\
\text { breech; if unstable lie, managed in labor }\end{array}$ \\
\hline Preterm labor & $\begin{array}{l}\text { Previable fetus delivered at birth center (included in mortality statistics); } \\
\text { viability until } 35 \text { weeks' gestation transferred to tertiary care hospital; } \\
35 \text { to } 37 \text { weeks' gestation transferred to local hospital }\end{array}$ \\
\hline Postdates & $\begin{array}{l}\text { Because of poor dating, no induction unless oligohydramnios (office } \\
\text { sonogram weekly starting } 41 \text { weeks); amniotic fluid documented as } \\
\text { "inadequate, adequate, or excessive" with induction recommended for } \\
\text { inadequate }\end{array}$ \\
\hline $\begin{array}{l}\text { Elective or social } \\
\text { induction }\end{array}$ & None \\
\hline Twins & Vaginal delivery offered unless twin A was not in vertex position \\
\hline $\begin{array}{l}\text { Labor dystocia with } \\
\text { normal maternal } \\
\text { and fetal status }\end{array}$ & $\begin{array}{l}\text { No cesarean until at least } 4 \text { hours of no cervical change in active labor } \\
\text { and on oxytocin }\end{array}$ \\
\hline Arrest of descent & $\begin{array}{l}\text { No cesarean unless no progress after pushing for more than } 3 \text { hours in } \\
\text { primipara and } 1-2 \text { hours in multipara mother; allowed to push even } \\
\text { longer if signs of progression and normal maternal and fetal status }\end{array}$ \\
\hline $\begin{array}{l}\text { Electronic fetal } \\
\text { monitoring }\end{array}$ & $\begin{array}{l}\text { None; instead, fetal status monitored using structured intermittent } \\
\text { auscultation }\end{array}$ \\
\hline $\begin{array}{l}\text { Vacuum and } \\
\text { forceps }\end{array}$ & No forceps; vacuum assist when indicated according to ALSO guidelines ${ }^{13}$ \\
\hline Labor pain & $\begin{array}{l}\text { Massage, hot packs, continuous clinician presence and encouragement; } \\
\text { husband present in all cases; no intravenous narcotics, no epidurals }\end{array}$ \\
\hline $\begin{array}{l}\text { Postpartum } \\
\text { hemorrhage }\end{array}$ & $\begin{array}{l}\text { Prevented or treated early by active management of } 3 \text { rd stage, one- } \\
\text { on-one nursing care with uterine assessment every } 5 \text { minutes in first } \\
\text { hour, aggressive intravenous fluid therapy, oxytocin, misoprostol, and } \\
\text { methylergonovine }\end{array}$ \\
\hline Oxytocin & $\begin{array}{l}\text { Used for labor induction, augmentation, active management of the 3rd } \\
\text { stage of labor, and treatment of postpartum hemorrhage }\end{array}$ \\
\hline Prenatal education & None beyond that taught at prenatal visits \\
\hline Discharge criteria & $\begin{array}{l}\text { Maternal stable vital signs, bleeding controlled; intravascular volume } \\
\text { replaced; infant stable. Minimum stay } 2 \text { hours; generally } 2 \text { to } 3 \text { hours }\end{array}$ \\
\hline
\end{tabular}


All fetal malpresentations were confirmed by ultrasound examination. Women wanting external cephalic version in the birthing center were advised that immediate surgical capabilities to address procedure complications were lacking, and verbal consent was obtained. Women with frank breech babies were allowed attempted vaginal delivery after a discussion that included risks of cord prolapse and head entrapment.

For women with twins, birthing center delivery criteria included first twin presenting in a vertex position and more than 37 weeks' gestation. Women were counseled on risks of a twin delivery without operative capabilities and offered hospital transfer.

Women in premature labor were appropriately counseled and offered transfer to a tertiary center if their pregnancy was less than 35 weeks or to VMH if their pregnancy was between 35 and 37 weeks; some refused. When a fetus was not viable or extremely preterm, patients generally preferred birthing center delivery. These infants are included in the mortality statistics.

No analgesics were administered during labor at the birthing center. Not using analgesics was the cultural norm, as was continuous support throughout labor.

Postpartum hemorrhage was prevented or treated early by active management of third-stage, one-on-one nursing care with uterine assessment every 5 minutes in the first hour, aggressive intravenous fluid therapy, oxytocin, misoprostol, and methylergonovine.

All women attending the birthing center were uninsured. They were charged $\$ 925$ per delivery regardless of length of stay or supplies used (except for Rh immune globulin, for which they were charged separately). The charge included a postpartum visit for mother and baby and the newborn screening tests.

We entered data from 1993 to 2010 birthing center records for all women admitted for labor into an Excel database for analysis. The birth records were written in a uniform format throughout the study period. The data were de-identified, and we converted dates into time periods to preserve confidentiality. This study was exempted by the University of Wisconsin Institutional Review Board.

Data entered included demographics, duration of stages of labor, transfer details, cesarean statistics, and perinatal outcomes. The denominator for rates (inclusion criteria) was all women admitted in labor to the birthing center, including those who planned to give birth there and those who were transferred in from home. For women who gave birth more than once at the birthing center, each pregnancy was included. Women who were transferred to a hospital before labor for conditions including those listed above were not counted when determining rates (exclusion criteria). Perinatal deaths were defined as the sum of fetal deaths plus neonatal deaths; fetal death was defined as any death beyond 20 weeks' gestation but before delivery, and neonatal death as death after birth but before 28 days of life.

We investigated birthing practices for the entire service area. By reviewing birthing center records for all deliveries between 2005 and 2009, we determined that $75 \%$ of women giving birth at the birthing center were from Vernon and Monroe counties. We then reviewed birth certificates for all Amish Vernon and Monroe county births between 2005 and 2009 at the Register of Deeds office, identified the birth location, and calculated the percentage of infants born at home, at the birthing center, at small local hospitals, and at tertiary hospitals.

We reviewed all VMH records of Amish births between 2005 and 2009 to determine whether women were referred from the birthing center or home and whether the referral was before or during labor.

\section{RESULTS}

Southwest Wisconsin Amish women give birth to most children at home. Of 1,178 births in Vernon and Monroe counties from 2005 to 2009, 683 (58\%) occurred in the home, 395 (34\%) occurred at the birthing center, 88 (7\%) took place in small community hospitals, mostly VMH, and 12 (1\%) occurred at tertiary hospitals with obstetricians on staff. Of VMH Amish births from 2005 through 2009, 41\% of mothers were transferred from the birthing center in labor, 29\% were transferred from attempted home births, 19\% were transferred from the birthing center antenatally, and $10 \%$ (5 patients) were self-referred to VMH for prenatal care and delivery.

From 1993 until 2010, 418 women were attended for 927 deliveries at the birthing center. Figure 1 shows the age and Figure 2 the parity of women in the study at the time of each delivery.

Ninety-six deliveries (10\%) were of women coming to the birthing center after planning a home birth (Table 2). This group accounted for $20 \%$ (7 of 35) of cesarean deliveries and 37\% (7 of 19) of perinatal deaths. Table 3 lists indications and frequency for transfers from the birthing center to the hospital. Transfer from the birthing center to the hospital occurred for $6 \%$ (48 of 834 ) of women planning go have their babies at the birthing center and 11\% (11 of 96) of women who transferred from home to the birthing center.

The overall cesarean rate was $4 \%$ (35 cesareans of 927 deliveries). The cesarean rate was $3 \%$ (28 of 834 
deliveries) for women planning a clinic birth and 7\% (7 of 96 deliveries) for women who transferred from home. Details of other cesarean deliveries are documented in Table 4.

The VBAC rate was $96 \%$ ( 88 of 92 deliveries). Of the TOLACs $93 \%$ (86 of 92 deliveries) took place with 36 women who had had 1 previous cesarean, $6 \%$ (6 of
92 deliveries) were of 2 women who had had 2 previous cesarean deliveries; no TOLACs were attempted for women with 3 or more prior cesarean deliveries.

Patients in labor with fetal malpresentation included those who failed or declined external version or who transferred to the birthing center without prenatal care. Of these women, 14 were primagravida and 8

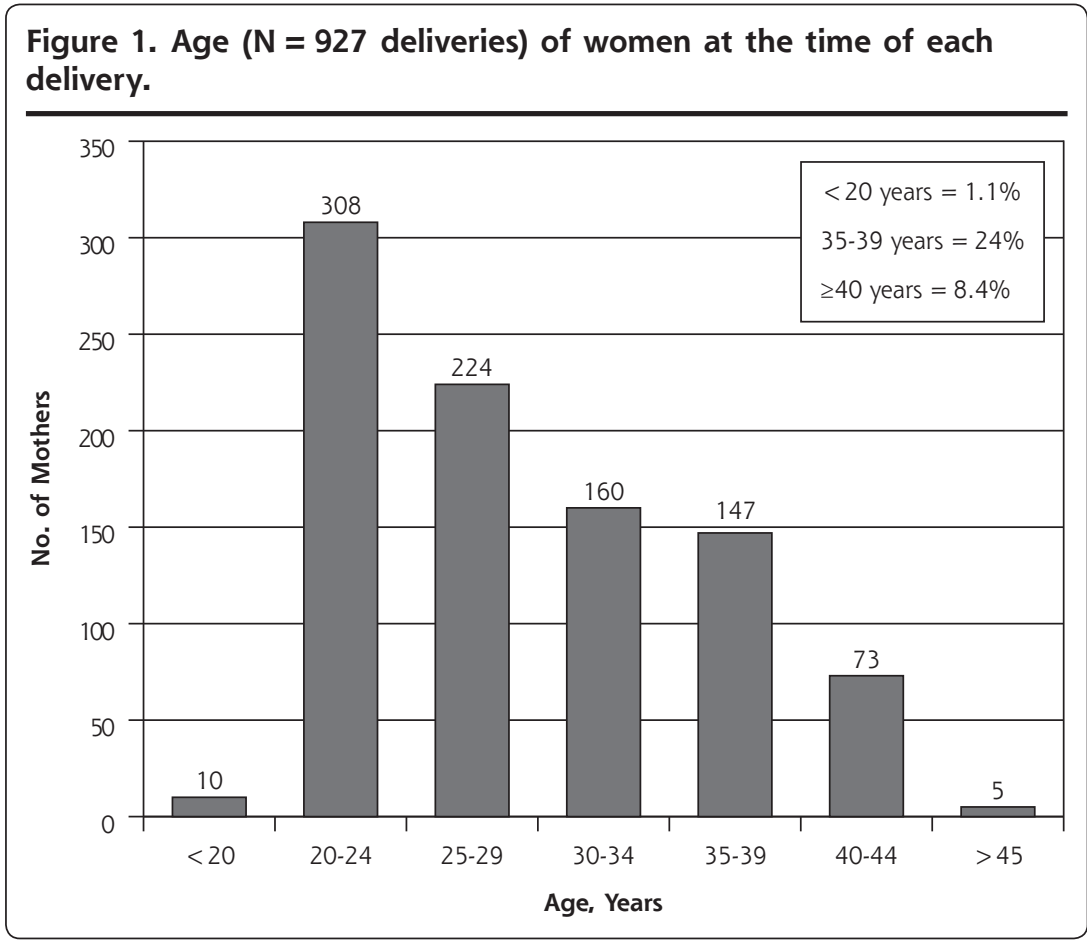

were multigravida with breech presentation. Two patients with frank breech presentations chose cesarean; 3 had nonfrank breech presentations, and cesarean delivery was performed. Three multiparous women had successful intrapartum version and vaginal delivery. Of the 14 remaining patients, all with frank breech presentation desiring trial of labor, 8 had a successful vaginal breech delivery, and 6 required cesarean for lack of descent. Seven of 8 successful breech presentations with vaginal delivery were in nulliparous patients.

Ten women had twins with the first baby in the vertex position; 1 mother was at 35 weeks' gestation and refused hospital transfer, 2 chose TOLAC, and 1 pregnancy was previable. Of the 9 sets of viable twins, 5 were in

\section{Figure 2. Parity ( $\mathrm{N}=927$ deliveries) of women at time of each delivery.}

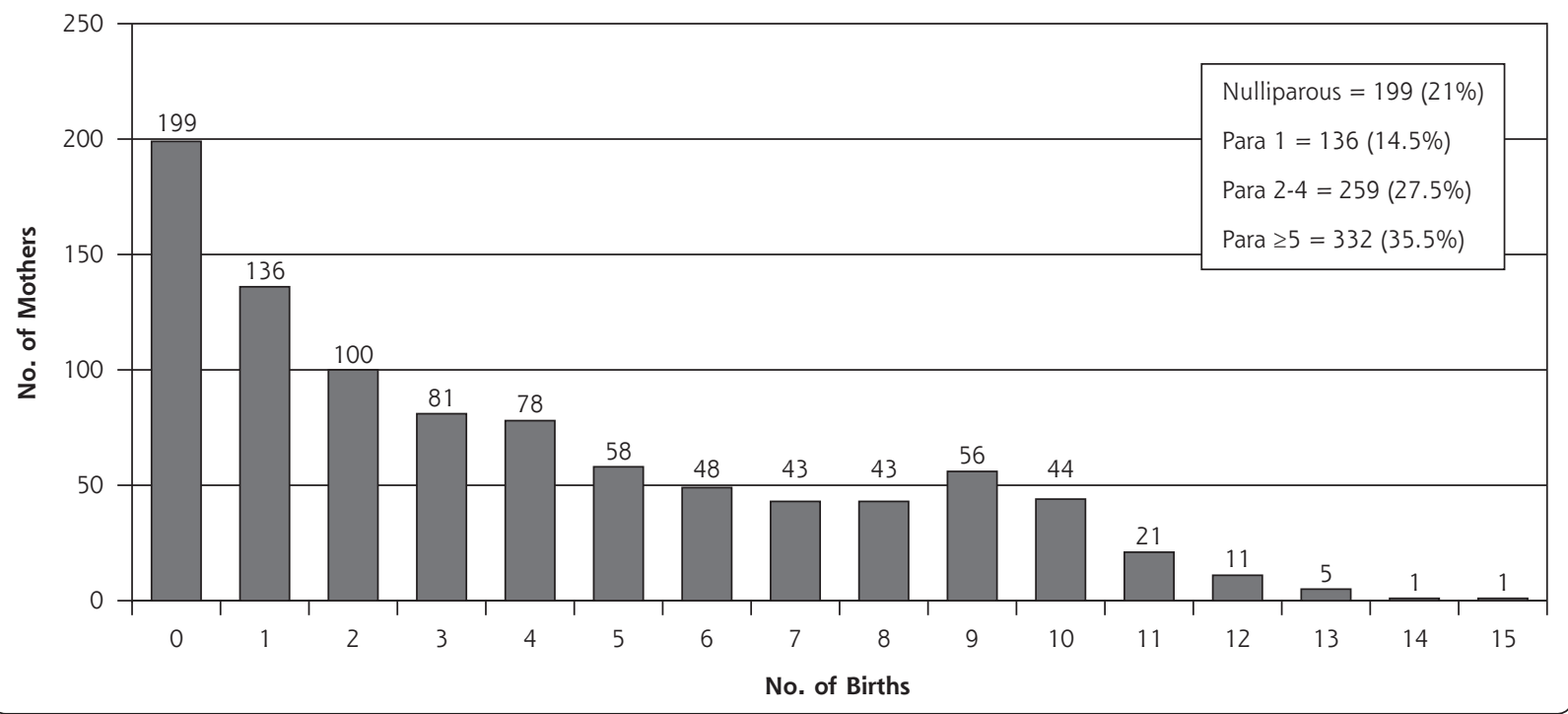


Table 2. Reason for and Number of Transfers From Home to Birthing Center or Hospital

\begin{tabular}{|c|c|c|c|}
\hline Reason & $\begin{array}{c}\text { Transfer to } \\
\text { and Delivery at } \\
\text { Birthing Center } \\
(\mathrm{n}=85)\end{array}$ & $\begin{array}{l}\text { Transfer to } \\
\text { and Delivery } \\
\text { at Hospital } \\
(n=11)\end{array}$ & $\begin{array}{c}\text { Total } \\
\text { Transfers } \\
(n=96)\end{array}$ \\
\hline Failure to progress & 29 & 2 & 31 \\
\hline Prolonged rupture of membranes & 16 & 1 & 17 \\
\hline Postdates with inadequate labor & 12 & 1 & 13 \\
\hline Midwife unavailable & 10 & 1 & 11 \\
\hline Malpresentations & 3 & 6 & 9 \\
\hline Absent or decreased fetal movement & 4 & 0 & 4 \\
\hline Third trimester bleeding & 4 & 0 & 4 \\
\hline Preterm labor & 2 & 0 & 2 \\
\hline Other ${ }^{\mathrm{a}}$ & 5 & 0 & 5 \\
\hline
\end{tabular}

a Bleeding vulvar varix; undiagnosed twins at 35 weeks' gestation, refused hospital transfer, delivered, but infants needed transfer and did well; arrived without notice or prenatal care; severe preeclampsia, refused hospital transfer; home birth canceled after husband's death.

and surgical complications during the pregnancies are outlined in Supplemental Table 1, available at http://annfammed.org/ content/10/6/530/suppl/DC1.

There were 14 fetal deaths (15.1 of 1,000 births) and 5 neonatal deaths ( 5.4 of 1,000 births) (see Supplemental Table 2, available at http://annfammed org/content/10/6/530/suppl/ DC1). Of the fetal deaths, 4 had multiple fetal anomalies, and 3 were previabile. Of the neonatal deaths, 2 were extremely premature, 1 had a genetic syndrome, 1 had multiple anomalies, and 1 had macrosomia and encephalopathy (but no dystocia and no neonatal

vertex/vertex and 3 were in vertex/breech positions. One woman gave birth to the first twin vaginally and had the second twin by cesarean delivery because of an arm presentation.

Maternal complications included 35 women with prolonged rupture of membranes, 4 with cord prolapse, 10 with preterm delivery, 18 with gestational hypertension, 1 with severe preeclampsia, 11 with shoulder dystocia, 42 with postpartum hemorrhage, 11 with placental abnormalities, and 4 with complex lacerations. Medical

\section{Table 3. Reason for Transfers From Birthing Center to Hospital}

\begin{tabular}{ll}
\hline Reason & No. \\
\hline Intrapartum transfers ( $=48$ ) & 25 \\
Malpresentation & 13 \\
Arrest of descent or dilation & 2 \\
Prolonged induction & 2 \\
Preterm & 2 \\
Third-trimester bleeding & 1 \\
Preeclampsia & 3 \\
Other & \\
Postpartum transfers of infant ( $\mathrm{n}=6$ ) & 1 \\
Anoxic encephalopathy (cord prolapse) & 1 \\
Jaundice (day 3 admission) & 1 \\
Preterm twins (35 wk), refused intrapartum transfer & 1 \\
Multiple anomalies & 1 \\
Encephalopathy with seizures (macrosomic infant) & 1 \\
Genetic syndrome with seizures and hypotonia & 2 \\
$\quad$ (2 siblings with same) & \\
Postpartum transfers of mother ( $\mathrm{n}=6$ ) & 3 \\
Postpartum hemorrhage (bleeding controlled but & \\
requiring transfusion) & 3 \\
Extensive perineal lacerations & 2 \\
\hline
\end{tabular}

hypoglycemia). Nine babies had a 5-minute Apgar of less than 7; 7 had no long-term issues, 1 has cerebral palsy, and 1 died of encephalopathy and seizures.

\section{DISCUSSION}

Our study shows that a low cesarean and high VBAC rate with good maternal and neonatal outcomes can be obtained in a nonhospital setting with skilled birth clinicians and basic resources. The Lafarge Birthing Center cesarean rate was $4 \%$ (35 of 927), the TOLAC rate was $100 \%$, and the VBAC rate was $95 \%$ (88 of 92). There were no cases of uterine rupture or maternal death. The risk of uterine rupture with TOLAC at

\section{Table 4. Characteristics of Cesarean Deliveries for All Lafarge Birthing Center Births}

\begin{tabular}{|c|c|c|}
\hline Characteristic & $\begin{array}{l}\text { No. of } \\
\text { Births }\end{array}$ & $\begin{array}{l}\text { Cesarean } \\
\text { Delivery } \\
\text { No. (\%) }\end{array}$ \\
\hline Total births & 927 & $35(4)$ \\
\hline \multicolumn{3}{|l|}{$\begin{array}{l}\text { Cesarean rate by planned delivery } \\
\text { location }\end{array}$} \\
\hline $\begin{array}{l}\text { Planned home delivery with intrapar- } \\
\text { tum transfer to birthing center }\end{array}$ & 96 & $7(7)$ \\
\hline Planned birthing center delivery & 834 & $28(3)$ \\
\hline \multicolumn{3}{|l|}{ Cesarean by gravidity } \\
\hline Primagravida & 199 & $15(8)$ \\
\hline Multigravida & 728 & $20(3)$ \\
\hline \multicolumn{3}{|l|}{ Indication for cesarean } \\
\hline Malpresentation & 35 & $17(49)$ \\
\hline Labor dystocia & 35 & $13(37)$ \\
\hline Cord prolapse & 35 & $3(9)$ \\
\hline $\begin{array}{l}\text { Failed induction with prolonged } \\
\text { rupture of membranes }\end{array}$ & 35 & $1(3)$ \\
\hline Failed induction for preeclampsia & 35 & $1(3)$ \\
\hline
\end{tabular}


term is 778 per $100,000,{ }^{2}$ so it is not unexpected that there would be no uterine ruptures in 92 TOLACs. The neonatal mortality rate of 5.4 of 1,000 was comparable to that of Wisconsin (4.6 of 1,000 in 2008) and the United States (4.5 of 1,000 in 2006). ${ }^{14}$

\section{Cesarean Rate}

Multiple cultural and intrapartum factors contributed to the low rate of cesarean delivery among women using the birthing center in this Amish community. These factors likely included infrequent inductions, active management of labor, external cephalic version, vaginal breech delivery, vaginal delivery of twins, encouragement of TOLAC, and continuous labor support without electronic fetal monitoring or epidural analgesia. The clinician mix and medicolegal climate were also quite different in the LaFarge Birthing Center, with community-based physicians and staff who knew the community and culture well and were respected for issues other than childbirth.

Multiparity, a low induction rate, and use of evidence-based guidelines for diagnosing failure to progress and arrest of descent contributed to the low rate (13 of 927) of cesarean delivery for labor dystocia. Elective induction was never offered, and postdates induction was recommended only if an ultrasound examination showed oligohydramnios. Allowing at least 4 (rather than 2) hours of adequate contractions with no cervical change before diagnosing failure to progress results in a lower cesarean rate with no worsening of maternal or fetal outcomes. ${ }^{15}$ In addition, during the second stage of labor, arrest of descent was not diagnosed so long as progress was being made and maternal and fetal status was reassuring. This approach has been documented in multiple studies ${ }^{16}$ to lower cesarean rates without compromising outcomes.

External cephalic version was offered by clinicians and accepted by women. The American Congress of Obstetricians and Gynecologists (ACOG) recommends "all women near term with breech presentations should be offered a version attempt."17 In non-Amish settings, external cephalic version is an underused option in the management of malpresentation. ${ }^{18}$

Vaginal breech delivery was offered for appropriate candidates. In the United States, vaginal breech delivery rates dropped precipitously after the 2000 Term Breech Trial ${ }^{19}$ showed increased neonatal mortality with vaginal breech delivery. Subsequent analysis 2 years later by the same authors found no neonatal or developmental risk with vaginal breech delivery, ${ }^{20}$ and ACOG changed its policy to again support vaginal breech delivery for appropriate candidates. ${ }^{21}$ The Society of Obstetricians and Gynecologists of Canada conclude that "careful case selection and labor manage- ment in a modern obstetrical setting may achieve a level of safety similar to elective Caesarean section." ${ }^{22}$ The PREMODA study of 5,579 cesarean and 2,526 planned vaginal deliveries for breech in Belgium and France found that $71 \%$ of women planning vaginal breech delivery were successful with no worsening of neonatal outcomes. ${ }^{23}$ Many clinicians, however, have abandoned the practice of vaginal breech delivery and now feel they do not have the experience to start offering this option again. ${ }^{21}$

Vaginal delivery was offered for full-term twins when the first twin was in a cephalic position. TOLAC for twin delivery is supported by ACOG. ${ }^{6}$ Although ACOG supports vaginal delivery of twins when the first twin is in the cephalic position, ${ }^{24}$ cesarean is frequent even for cephalic presentation of twins in many hospitals.

Clinician experience may be a factor. All deliveries were attended by a family physician or nurse midwife. Clinician mix was identified as a reason for lower cesarean rates in Indian Health Service (IHS) studies. ${ }^{25-27}$ In Zuni-Ramah Hospital, more than $95 \%$ of births were attended by family physicians. ${ }^{25}$ At the Santa Fe Indian Hospital, $82 \%$ of births were attended by nurse midwives, and those attended by obstetricians had an increased risk of cesarean (odds ratio $=2.4 ; P=.02$ ) after controlling for medical and obstetric factors. ${ }^{26}$ Tuba City cited a high percentage of deliveries by midwives as a reason for its lower cesarean rate. ${ }^{27}$

Financial incentives for cesarean delivery may affect cesarean rates in certain systems. ${ }^{28}$ At the LaFarge Birthing Center, there was no financial incentive for operative delivery. Additionally, malpractice concerns may increase non-Amish cesarean rates. In one survey, $29 \%$ of obstetricians reported that malpractice concerns have caused them to increase the number of cesareans they perform. ${ }^{2}$ Most Amish do not believe in litigation and trust clinicians willing to work with their beliefs about childbirth and work in an uninsured, cash economy. ${ }^{11}$

Finally, and importantly, cultural beliefs and history are important factors in what women expect in the birthing process. Amish often prefer out-of-hospital and low-technology births for reasons including "reduced cost, increased comfort and privacy and a chance for a 'more natural birth."'11 These beliefs make it easier for clinicians caring for Amish women to follow evidencebased guidelines and avoid unnecessary surgery.

\section{Higher VBAC Rate}

In our study, $96 \%$ of births after a previous cesarean were vaginal, in sharp contrast with the general US rate of $8 \%$ from $2006 .{ }^{2}$ The birthing center's high VBAC rate contributes substantially to its low overall 
cesarean rate. The higher VBAC rates in IHS hospitals and the LaFarge Birthing Center likely have to do with clinicians and patients.

Although the clinicians in the LaFarge Birthing Center universally offer TOLAC to women who had a previous low-transverse cesarean, many clinicians elsewhere do not. ${ }^{2}$ Even when TOLAC is offered, it may be presented in a way that guides women not to choose it ${ }_{i}$ "several studies suggest that how risk is presented and communicated by providers may have a powerful effect." ${ }^{\prime 2}$

The high VBAC rate of success in this study was achieved not only by support of VBAC by the attending clinicians but also by a high acceptance of TOLAC by the women. Although some women wanting an elective repeat cesarean delivery may have self-referred to a hospital, no woman who came to the birth center chose a repeat cesarean over a TOLAC. In other populations, a much higher percentage of women who are offered a TOLAC choose a cesarean delivery. Desire for a partner's involvement, a sense that vaginal birth can be empowering, improved maternal-infant bonding, greater ease with breastfeeding, and expectation of an easier recovery have all been identified as reasons that women choose a TOLAC. ${ }^{2}$ These reasons can be promoted in office visits, public service announcements, and other venues in changing cultural attitudes toward greater support for VBAC.

Labor support may also be a factor in the low cesarean rate at the LaFarge Birthing Center. A Cochrane Review of 21 trials involving 15,061 women found that women with continuous support in labor were less likely to have a cesarean delivery, instrumental vaginal birth, or a baby with a low 5-minute Apgar score. ${ }^{29}$ At the birthing center, the physician, midwife, or nurse was at the mother's bedside throughout each labor.

\section{Maternal-Fetal Outcomes}

The positive outcomes cannot be explained by low-risk populations. Women giving birth at the LaFarge Birthing Center were at higher risk than the general US population in terms of higher rates of advanced maternal age and grand multiparity (Figures 1 and 2), as is the case in other Amish communities. ${ }^{12}$

As in IHS hospitals, deliveries in our study resulted in a low primary cesarean rate and a high VBAC rate. At $4 \%$, our cesarean rate is much lower than the national rate of $33 \%{ }^{2}$ and closer to the rates of $7.3 \%,{ }^{25} 9.6 \%,{ }^{26}$ and $13.5 \%{ }^{27}$ from IHS hospitals. The VBAC rate of $95 \%$ is much higher than the national rate of $8.5 \%{ }^{6}$

\section{National Birth Center Standards}

Subsequent to the National Birth Center Study (NBCS), many birthing centers and organizations developed policies of not attempting VBACs. ${ }^{30}$ The NBCS of 1,913 women attempting VBAC in birthing centers found 6 uterine ruptures $(0.4 \%), 1$ hysterectomy $(0.1 \%), 15$ infants with a 5 -minute Apgar of less than $7(1.0 \%)$, and 7 fetal deaths $(0.5 \%){ }^{30}$ The study concluded that "birth centers should refer women who have undergone previous cesarean deliveries to hospitals for delivery."${ }^{130}$ In the LaFarge community, most women with a previous cesarean refuse hospital delivery. TOLAC is offered at the birthing center as a safer alternative to TOLAC at home. It is uncertain whether any of the perinatal deaths or other bad outcomes could have been avoided if the babies had been delivered in a hospital rather than a birthing center. Without the birthing center, mothers would probably would have given birth at home rather than the hospital, without a physician or nurse in attendance.

We do not argue that TOLAC in birthing centers should be adopted for the general US population. We do believe our experience offers important lessons that, in the right circumstances and practice environment, cesarean rates can be decreased and VBAC rates increased in a safe and evidence-based manner. The LaFarge Birthing Center does offer a model of care sensitive to cultural norms that highly value the health of the childbearing woman.

To read or post commentaries in response to this article, see it online at http://www.annfammed.org/content/10/6/530.

Key words: birthing centers; Wisconsin; prenatal care; cesarean section; pregnancy; natural childbirth

Submitted June 2, 2011; submitted, revised, January 20, 2012; accepted February 20, 2012

Previous presentations: Findings were presented at a poster session at the Annual Spring Conference of the Society of Teachers of Family Medicine, April 27-May 1,2011, New Orleans, Louisiana.

\section{References}

1. Wall E, Roberts R, Deutchman M, Heston W, Atwood L, Ireland B. Trial of Labor After Cesarean (TOLAC), Formerly Trial of Labor Versus Elective Repeat Cesarean Section for the Woman With a Previous Cesarean Section: American Academy of Family Physicians; 2005. http:// www.annfammed.org/content/suppl/2005/07/26/3.4.378.DC1/ TOLAC_2005_Guideline.pdf.

2. National Institutes of Health Consensus Development Conference Panel. National Institutes of Health Consensus Development conference statement: vaginal birth after cesarean: new insights March 8-10, 2010. Obstet Gynecol. 2010;115(6):1279-1295.

3. Hamilton B, Martin J, Ventura S. Births: Preliminary Data for 2009. National Statistics Reports. Vol 59. Hyattsville, MD: National Center for Health Statistics; 2010.

4. Solheim KN, Esakoff TF, Little SE, Cheng YW, Sparks TN, Caughey $A B$. The effect of cesarean delivery rates on the future incidence of placenta previa, placenta accreta, and maternal mortality. J Matern Fetal Neonatal Med. 2011;24(11):1341-1346. 
5. Martin JA, Hamilton BE, Sutton PD, et al. Births: final data for 2007. Natl Vital Stat Rep. 2010;58(24):1-85.

6. American College of Obstetricians and Gynecologists. ACOG Practice bulletin no. 115: Vaginal birth after previous cesarean delivery. Obstet Gynecol. 2010;116(2 Pt 1):450-463.

7. Healthypeople.gov. Maternal Infant and Child Health. Healthy People 2020 topics and objectives. http://healthypeople.gov/2020/ topicsobjectives2020/objectiveslist.aspx?topicid=26. Accessed Nov 6, 2011.

8. Appropriate technology for birth. Lancet. 1985;2(8452):436-437.

9. Zhang J, Troendle J, Reddy U, et al. Contemporary cesarean delivery practice in the United States. Am J Obstet Gynecol. 2010;203(4): 326.e1-326.e10.

10. Adams CE, Leverland MB. The effects of religious beliefs on the health care practices of the Amish. Nurse Pract. 1986;11(3):58, 63, 67.

11. Campanella K, Korbin JE, Acheson L. Pregnancy and childbirth among the Amish. Soc Sci Med. 1993;36(3):333-342.

12. Acheson LS. Perinatal, infant, and child death rates among the Old Order Amish. Am J Epidemiol. 1994;139(2):173-183.

13. Advanced Life Support in Obstetrics (ALSO) Syllabus. 4th ed. Leawood, KS: American Academy of Family Physicians; 2011.

14. Wisconsin Department of Health Services DoPH, Office of Health Informatics. Wisconsin Births and Deaths 2008. November 2009.

15. Rouse DJ, Owen J, Hauth JC. Active-phase labor arrest: oxytocin augmentation for at least 4 hours. Obstet Gynecol. 1999;93(3): 323-328.

16. Shields SG, Ratcliffe SD, Fontaine P, Leeman L. Dystocia in nulliparous women. Am Fam Physician. 2007;75(11):1671-1678.

17. American College of Obstetricians and Gynecologists. ACOG Practice Bulletin, Number 13. External Cephalic Version. Obstet Gynecol 2000.

18. Coco AS, Silverman SD. External cephalic version. Am Fam Physician. 1998;58(3):731-738, 742-744.

19. Hannah ME, Hannah WJ, Hewson SA, Hodnett ED, Saigal S, Willan AR; Term Breech Trial Collaborative Group. Planned caesarean section versus planned vaginal birth for breech presentation at term: a randomised multicentre trial. Lancet. 2000;356(9239):1375-1383.
20. Whyte H, Hannah ME, Saigal S, et al; Term Breech Trial Collaborative Group. Outcomes of children at 2 years after planned cesarean birth versus planned vaginal birth for breech presentation at term: the International Randomized Term Breech Trial. Am J Obstet Gynecol. 2004;191(3):864-871.

21. ACOG Committee on Obstetric Practice. ACOG Committee Opinion No. 340. Mode of term singleton breech delivery. Obstet Gynecol. 2006;108(1):235-237.

22. Kotaska A, Menticoglou S, Gagnon R, et al; Society of Obstetricians and Gynaecologists of Canada. SOGC clinical practice guideline: Vaginal delivery of breech presentation: no. 226, June 2009. Int J Gynaecol Obstet. 2009;107(2):169-176.

23. Goffinet F, Carayol M, Foidart JM, et al; PREMODA Study Group. Is planned vaginal delivery for breech presentation at term still an option? Results of an observational prospective survey in France and Belgium. Am J Obstet Gynecol. 2006;194(4):1002-1011.

24. American College of Obstetricians and Gynecologists Committee on Practice Bulletins-Obstetrics; Society for Maternal-Fetal Medicine; ACOG Joint Editorial Committee. ACOG Practice Bulletin \#56: Multiple gestation: complicated twin, triplet, and high-order multifetal pregnancy. Obstet Gynecol. 2004;104(4):869-883.

25. Leeman L, Leeman R. A Native American community with a $7 \%$ cesarean delivery rate: does case mix, ethnicity, or labor management explain the low rate? Ann Fam Med. 2003;1(1):36-43.

26. Mahoney SF, Malcoe LH. Cesarean delivery in Native American women: are low rates explained by practices common to the Indian health service? Birth. 2005;32(3):170-178.

27. Lessons at Indian hospital about births. New York Times. March 6, 2010. http://www.nytimes.com/2010/03/07/health/07birth.html. Accessed Nov 6, 2011.

28. Keeler EB, Brodie M. Economic incentives in the choice between vaginal delivery and cesarean section. Milbank Q. 1993;71(3): 365-404.

29. Hodnett ED, Gates S, Hofmeyr GJ, Sakala C, Weston J. Continuous support for women during childbirth. Cochrane Database Syst Rev. 2011;(2):CD003766

30. Lieberman E, Ernst EK, Rooks JP, Stapleton S, Flamm B. Results of the national study of vaginal birth after cesarean in birth centers. Obstet Gynecol. 2004;104(5 Pt 1):933-942. 